\title{
Methodology for the Water Injection System Design Based on Numerical Models
}

\author{
Miroslav Spodniak ${ }^{1}$, Ladislav Főző ${ }^{1}$, Rudolf Andoga ${ }^{2}$, \\ Karol Semrád ${ }^{1}$, Károly Beneda ${ }^{3}$
}

${ }^{1}$ Department of aviation engineering, Faculty of Aeronautics of Technical University of Košice, Rampová 7, 04001 Košice, Slovakia

miroslav.spodniak@tuke.sk, ladislav.fozo@tuke.sk,karol.semrad@tuke.sk

${ }^{2}$ Department of avionics, Faculty of Aeronautics of Technical University of Košice, Rampová 7, 04001 Košice, Slovakia

rudolf.andoga@tuke.sk,

${ }^{3}$ Department of Aeronautics, Naval Architecture and Railway Vehicles, Faculty of Transport Engineering and Vehicle Engineering, Budapest University of Technology and Economics, Müegyetem rkp. 3, H-1111 Budapest, Hungary kbeneda@vrht.bme.hu

\footnotetext{
Abstract: Jet engines are nowadays one of the most popular ways of propulsion for aircraft. This type of propulsion is widely used also in other sectors of industry. The main challenging task for designers is to design reliable and also powerful propulsion units. There are many manners for increasing the thrust of the particular jet engine and one of them is water injection into the compressor. In order to design such a system, it is necessary to have information about the flow parameters in the compressor. The proposed article deals with the CFD modeling of the radial compressor in order to estimate flow parameters for further research in the field of increasing thrust. One of the aims of the article is to develop a numerical model of the compressor and carry out the numerical analysis using CFD software ANSYS CFX. The analysis can be in further research performed multiple times for multiple regimes and results can be compared with the experimental measurements of thermodynamical values if the proper CFD model is developed. The main target of the paper is to establish the methodology for the amount of water estimation for particular engines. The methodology is introduced in the third chapter of the paper. Following the CFD model presented in the article and the methodology for water amount estimation, further research is presented in the fourth chapter of the article.
}

Keywords: temperature model; pressure model; CFD simulation; water injection system; centrifugal compressor 


\section{Introduction}

Centrifugal compressors are nowadays extensively used in an area of applications including small jet engines, turboprop engines, power generators, and auxiliary power units, air conditioning, etc. [1] The construction of this device is an important factor in terms of the performance and efficiency of the system. Centrifugal compressors have to meet some specific requirements to ensure reliable engine operation, such as reliable work during the particular phases of the flight, durability, resistance to high loads. [2,1] Jet engines have to also provide enough thrust during their operation for the specific phases of the flight. [1] There are some special cases when a range of phenomena is occurring, including noise generation, tip clearance, surge, and unsteady flow and, also the compressor performance. In order to investigate the mentioned processes numerical modeling using the CFD method is a convenient tool. CFD modeling is not the only appropriate way to estimate characteristics of the compressors that are already made but also for the modeling flow during the design phase of the centrifugal compressors. An important aspect of the numerical calculation process is evaluating the results according to the experimental data [2,3].

Centrifugal compressor is a dynamic axisymmetric machine, which achieves a pressure rise by adding kinetic energy or velocity to a continuous flow of fluid through the impellers. Apart from the pressure rise in the impeller, the kinetic energy is converted to increase the pressure using the diffuser that is decreasing the flow speed and increasing the pressure [4].

The thrust of the jet engine is one of the fundamental parameters and is defined as the force to be the change in momentum of an object with a change in time. Momentum is the object's mass times the velocity. [2] There are some ways for increasing the thrust and one of them is water injection into the compressor inlet of the engine. This principle has been well known for many years, so it is possible to implement the methodology for a particular engine. In order to design a water injection system, the thermodynamic parameters of the system should be well known, therefore, in this study, the flow parameters of the centrifugal compressor will be estimated. According to the flow parameters obtained from the CFD simulation, the methodology for the water injection system will be established [4]. In [4] different parameters of the centrifugal compressor were estimated using CFD analysis in ANSYS, also the impact of the flow on the compressor operation and phenomena were studied in [4]. Using a similar methodology the 3D modeling of the flow in the compressor is carried out. [6, 7].

There is a large number of centrifugal compressor types, investigated centrifugal compressor of the iSTC-21v jet engine consists of an inlet, impeller, diffuser, and the casing. [8] The compressor hub with impeller and stator vanes is shown in Figure 1 . The model is partially modified for the CFD analysis. The impeller is semi-enclosed with 20 blades. Research is based on the data of iSTC-21v engine 
but the methodology is also applicable on TKT-1 turbojet (which is almost the same as iSTC-21v). The TKT-1 is a single spool, centrifugal compressor, axial turbine jet engine, which is equipped with an annular combustion chamber $[9,10]$.

The object of the CFD study is the compressor section with the inlet. The inlet of TKT-1 got a vertical cylindrical air inlet duct due to the testbed location [9] and that is the main difference in comparison with iSTC-21v engine. Such an inlet allows air inlet from the undisturbed regions and minimizes foreign object damage possibilities. The Centrifugal compressor with the diffuser is presented in Fig. 1. The 3D model of the compressor has to be modified for the CFD analysis [9, 1].
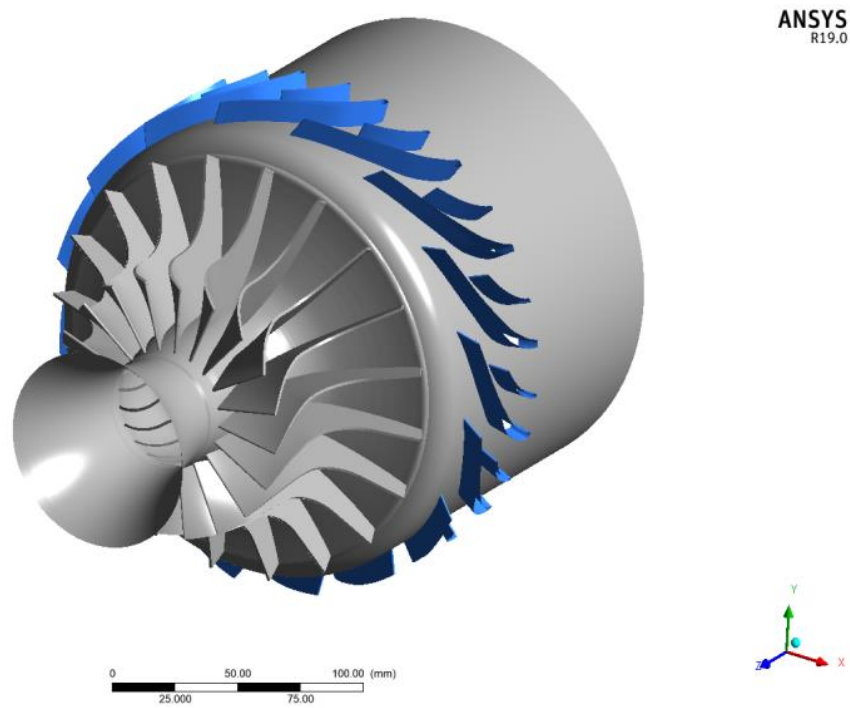

Figure 1

The geometry of the compressor TKT-1

\section{Numerical Study}

Currently, 3D virtual modeling is popular among designers due to its advantages, for instance, in the proposed study CFD modeling is an effective tool in order to estimate flow parameters. For the study, the ANSYS R19.0 software is used to carry out a steady-state analysis. Centrifugal compressor analysis involves the rotating flow domain. In ANSYS, the flow features associated with this problem can be also analyzed using the multiple reference frame capability. The analysis of the problem is including stationary and dynamic parts, compressor stator, and impeller, so the interaction of the parts is described in the following lines. 
The analyses between the stationary and dynamic parts as the centrifugal compressor also often involves the examination of the transient effects. Transient effects are caused due to flow interaction between the rotor and stator parts. In the study, the sliding mesh capability of ANSYS is used to compute the transient flow in a centrifugal compressor. The interaction between the rotor and stator is modeled by allowing the mesh which is associated with the rotor to rotate relative to the stationary mesh associated with the stator part of the compressor $[10,11$, 12].

In the presented paper the Singe Phase study is performed, which means the water is not included yet. In this type of study ANSYS fluent solves for an arbitrary scalar $\Phi_{k}$ the equation:

$$
\frac{\partial \rho \phi_{k}}{\partial t}+\frac{\partial}{\partial x_{i}}\left(\rho u_{i} \phi_{k}-\Gamma_{k} \frac{\partial \phi_{k}}{\partial x_{i}}\right)=S_{\phi_{k}} k=1, \ldots, N
$$

Where $\Gamma_{k}$ and $s_{\Phi_{k}}$ are the diffusion coefficient and source term you supplied for each of the $N$ scalar equations. Note that $\Gamma_{k}$ is defined as a tensor in the case of anisotropic diffusivity. The diffusion term is, therefore $\nabla .\left(\Gamma_{k} . \Phi_{k}\right)$. For isotropic diffusivity, $\Gamma_{k}$ could be written as $\Gamma_{k} I$ where I is the identity matrix. For the steadystate case, ANSYS Fluent will solve one of the three equations, depending on the method used to compute the convective flux $[10,11]$.

ANSYS compute the following equation in the case that the flux is not concluded:

$$
-\frac{\partial}{\partial x_{i}}\left(\Gamma_{k} \frac{\partial \phi_{k}}{\partial x_{i}}\right)=S_{\phi_{k}} k=1, \ldots, N
$$

where $\Gamma_{k}$ and $s_{\Phi_{k}}$ are the diffusion coefficient and source term you supplied for each of the 5 scalar equations. If the convective flux is to be computed with a mass flow rate, ANSYS Fluent will solve the equation:

$$
\frac{\partial}{\partial x_{i}}\left(\rho u_{i} \phi_{k}-\Gamma_{k} \frac{\partial \phi_{k}}{\partial x_{i}}\right)=S_{\phi_{k}} k=1, \ldots, N
$$

The software ANSYS is working as most CFD software solutions based on the Navier-Stokes equations for viscous flow. These consist of the continuity equation, the momentum equation, and the energy equation (2). The equations are reflecting the changes in flow in each solution element of computational mesh [10, $11,12]$. Thermodynamics parameters can be estimated also analytically one of the methods is represented by the following formulas. The pressure in the inlet can be assumed as follows: 


$$
p_{0 t}=p_{0} \cdot\left(1+\frac{K-1}{2} \cdot M_{0}^{2}\right)^{\frac{K}{K-1}}
$$

Where $p_{0}$ is the atmospheric pressure, $\boldsymbol{K}$ is the adiabatic exponent and $\mathrm{M}$ is the Mach number $[10,11,12]$. The temperature in the inlet can be estimated according to the formula:

$$
T_{0 t}=T_{0 \cdot}\left(1+\frac{\kappa-1}{2} \cdot M_{0}^{2}\right)
$$

The $\mathrm{T}_{0}$ is the atmospheric temperature. The pressure at the inlet of the impeller can be estimated by multiplying formula (4) by the pressure retention factor [10]. After modifying the formulas we can writhe the formula for the temperature at the outflow of the compressor as:

$$
T_{2}=T_{2 t}-\frac{c_{\Omega}^{a}}{2 \cdot c_{p}}
$$

Where $T_{2 t}$ is the temperature at the inlet of the bladeless diffusor of the compressor, $\mathrm{c}_{4}$ is the speed at this point and $\mathrm{cp}$ is the heat capacity [11]. Then the overall pressure at the outflow of the compressor can be estimated according to the formula:

$$
p_{2}=p_{2 t} \cdot\left(\frac{T_{2}}{T_{1}}\right)^{\frac{n_{1}}{n_{1}-1}}
$$

$\mathrm{p}_{2}$ states for the overall pressure at the outlet of the compressor and $\mathrm{p}_{2 t}[12]$. The formulas are showing one of the ways for calculation thermodynamical parameters, which are essential for the methodology described in Section 3. However, the results would be not precise due to calculation only in a particular point that is why CFD methods for calculation are used. The model is described in the following chapters also this model will be used in Chapter 3.

\subsection{CFD Modeling of Centrifugal Compressor}

The object of an investigation is axisymmetric assembly, which means that the numerical model can be divided according to the symmetry into 20 symmetric parts. The model represents single-stage radial compressor consists of two blade row - rotor blades and stator vanes. The geometry (Figure 1) is adapted for the cyclic symmetry analysis as the case of this study, thus one sector is created for both components stator and rotor.

The computational domain consists of the inlet, rotating impeller, and stationary diffuser with frozen rotor interfaces between adjacent domains. The rotational speed of the impeller is set according to the experimental setup. 
M. Spodniak et al.

Methodology for Water Injection System Design Based on Numerical Models

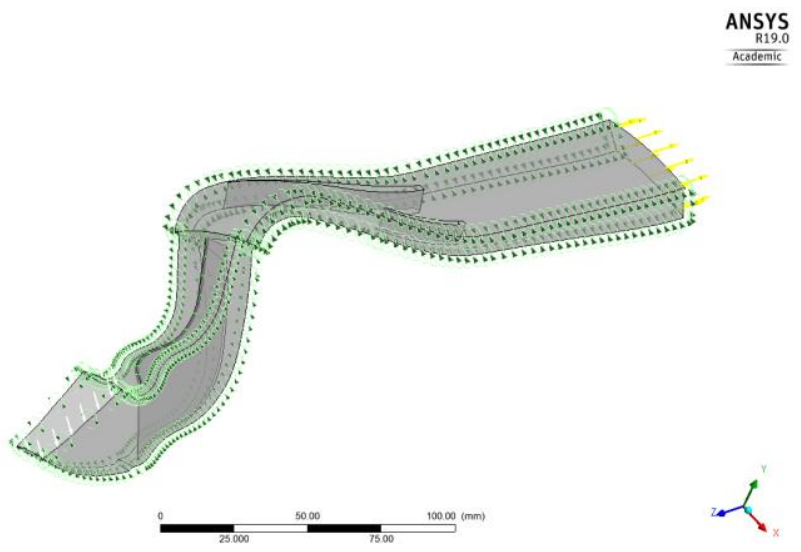

Figure 2

Computational domain of the compressor

2.2 Boundary Conditions

Boundary conditions are clearly seen in Figure 2, the temperature and pressure are applied as the boundary condition at the inlet interface. Between the adjacent blade passages, the periodic boundary condition is applied. The study is performed for teen different regimes according to the measured data (Figure 5). For instance one of them $3^{\text {rd }}$ regime is presented, where boundary conditions are defined by the inlet pressure and temperature at $39960 \mathrm{rpm}$.

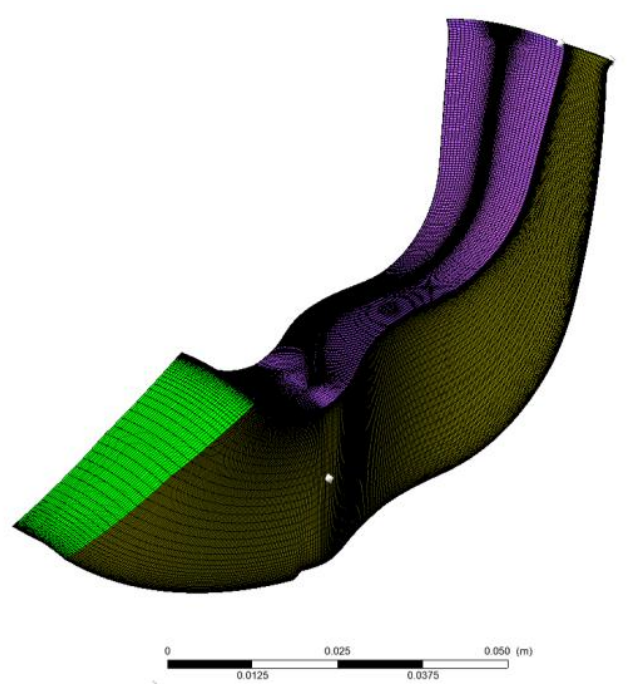

Figure 3
ANSYS

R19.0

Radial compressor mesh

$-52-$ 
The mesh is created in the TurboGrid software. For mesh creation, it is essential to have some information about the system. Such information includes the location of the geometry files for all components (hub, shroud, and blades) the mesh topology type, and the distribution of mesh nodes. One of the crucial parts of the meshing process is the preparation of the geometry. Hub, shroud, and the blades have to be imported to TurboGrid software as coordinates with the axis of rotation Z. Subsequently, the geometry is generated in the software and the meshing process can be started. Once the geometry is defined, the next step is to create the topology that guides the mesh. Afterward the topology creation number of layers for both parts is defined. The mesh is created using the same ideology for both components stator and rotor and consists of the hexahedral elements. Mesh is represented by Figures 3 and 4. For the boundary condition application, ANSYS CFX software is selected, which is a highly accurate solver for robust solutions such as compressor assembly $[10,11,12]$.

The mesh is generated for both components stator and roto separately and during the preprocessor process, the computational domain from the two parts is created.

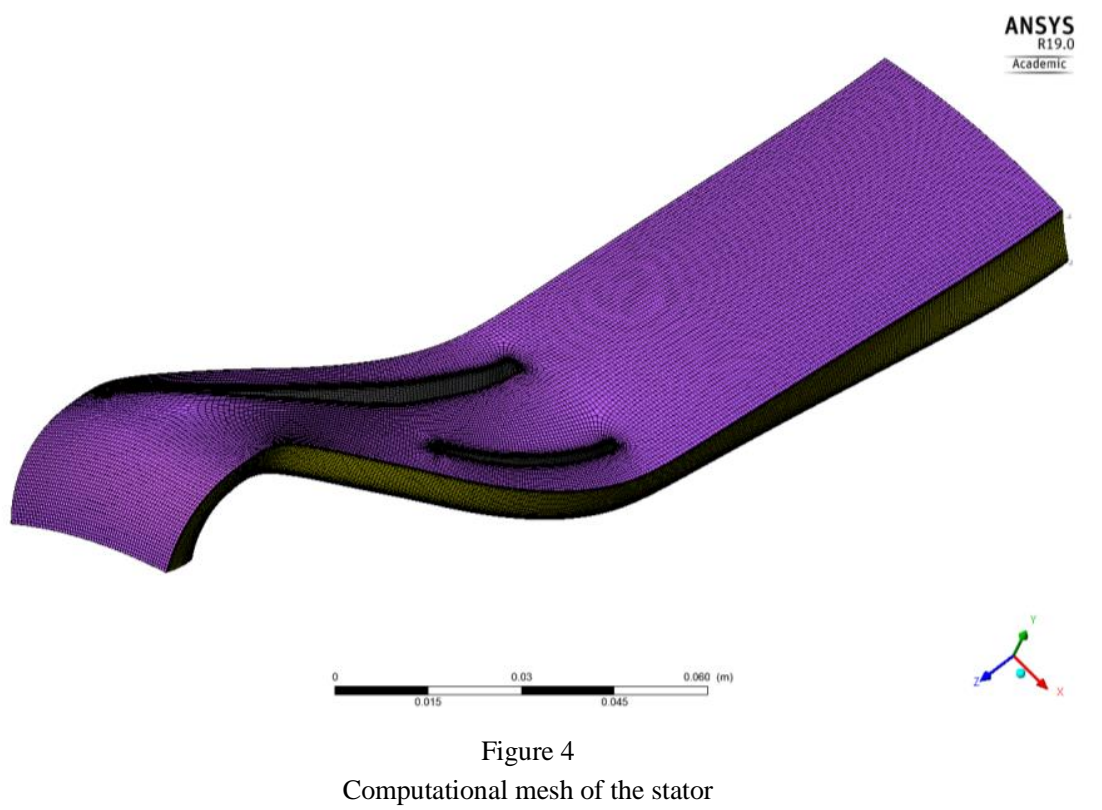

The mesh of the stator consists of 927405 elements and the rotor of the centrifugal compressor consists of 1055168 elements.

The analysis is performed for multiple regimes according to the measured data and for instance, in the proposed study the third regime is presented. The rpm (Figure 5), temperature, and pressure in the compressor section are measured. The boundary conditions are set according to the measured data in front of the centrifugal compressor. In Figure 5 the rpm for one run is measured using this 
data it is possible to simulate multiple regimes of centrifugal compressor operation by CFD methods [13].

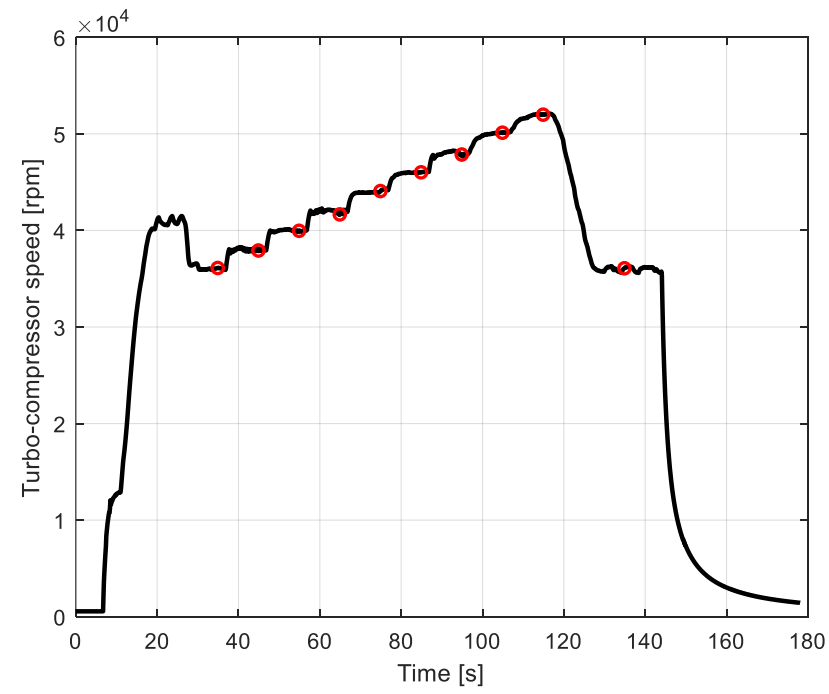

Figure 5

Measured data of iSTC-21v jet engine

In the presented study one regime is taken into an account in terms of CFD simulation. The third regime is simulated in ANSYS CFX software with 39960 $\mathrm{rpm}$ and the measured pressure at this operating point is $276414,6 \mathrm{~Pa}$.

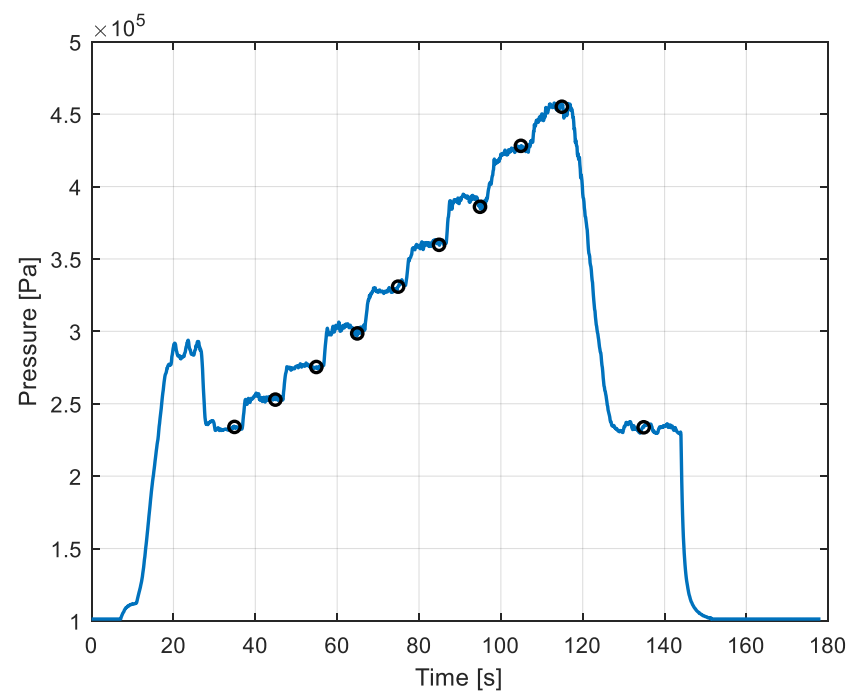

Figure 6

Measured pressure of iSTC-21v jet engine 


\subsection{Results of CFD Analysis}

There are few most important parameters of the thermodynamic cycle of a jet engine that have a significant impact on the performance of the engine. During the analysis, the goal is to monitor mainly the temperature and pressure. These two parameters will be used for water injection methodology, which will be described in Chapter 3.

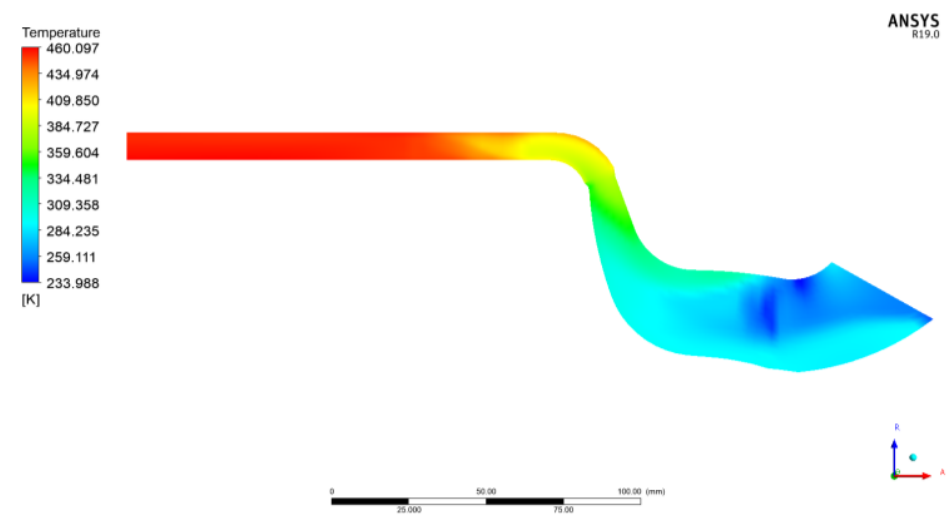

Figure 7

Temperature map of the compressor in meridional plane

The temperature is one of the crucial aspects when the thermodynamic cycle of the engine is investigated. The temperature field computed using CFX is shown in Figure 7. The results are comparable with the measurement of the temperature in iSTC-21v jet engine. The second estimated thermodynamical parameter during the CFD simulation is the pressure at the outflow of the compressor. Maximal pressure during the simulated regime of the engine is $277000 \mathrm{~Pa}$, which is in the comparison with the measurement of highly accurate results (Figure 8).
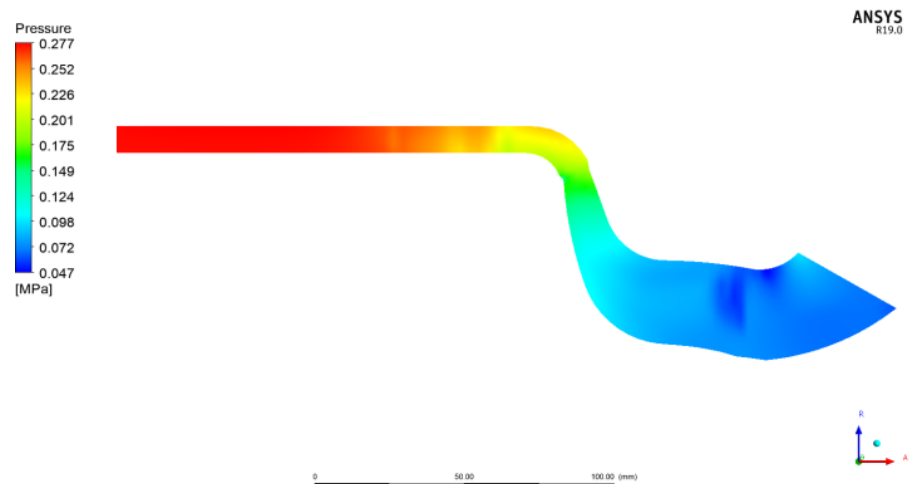

Figure 8

Pressure field of the compressor in meridional plane 
In Figure 9 the Mach Number is presented on the blade to blade plot. The plot shows an increasing trend of Mach number from inlet to the impeller and in the diffusor area the Mach Number is decreased, according to the theory.

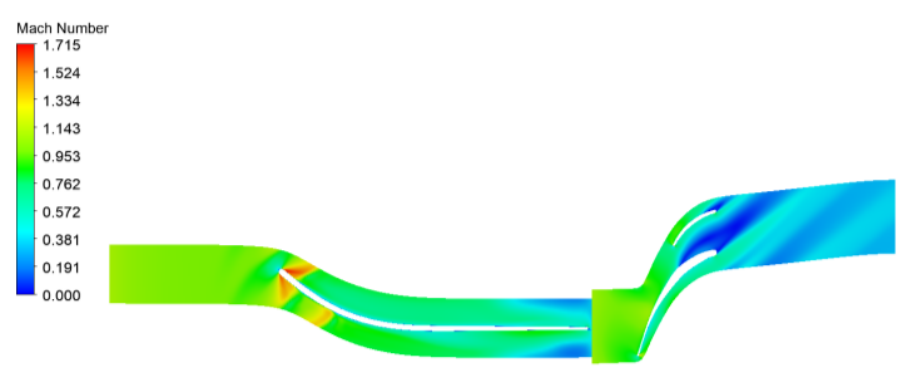

Figure 9

Mach Number of the compressor

Model is created for 10 regimes of the compressor operation, for instance, the comparison for the first four runs is in Table 1. The estimated temperature and pressure are compared using CFD methods. According to the results it can be clearly seen that the accuracy of the models is high and models of temperature and pressure will be used in the future while water injection system designing. Results for the rest regimes are in a similar range.

Table 1

Results Comparison of the Measured and Estimated Data

\begin{tabular}{|c|c|c|c|c|c|c|}
\hline Run & $\begin{array}{l}\text { Measured } \\
\text { Temp. }\left[{ }^{\circ} \mathrm{C}\right]\end{array}$ & \multicolumn{1}{l}{$\begin{array}{l}\text { Measured } \\
\text { Pressure }\end{array}$} & \multicolumn{1}{l}{$\begin{array}{l}\text { Temp. } \\
{\left[{ }^{\circ} \mathrm{C}\right] \text { CFD }}\end{array}$} & \multicolumn{1}{l}{$\begin{array}{l}\text { Pressure } \\
\text { CFD }\end{array}$} & \multicolumn{1}{l}{$\begin{array}{l}\text { Error } \\
\text { Temp. [\%] }\end{array}$} & $\begin{array}{l}\text { Error } \\
\text { P. }[\%]\end{array}$ \\
\hline 1 & 423,8 & 234000 & 421,4 & 237100 & 0,566 & 1,325 \\
\cline { 2 - 7 } 2 & 427,3 & 252800 & 425,5 & 251110 & 0,421 & 0,669 \\
\cline { 2 - 7 } 3 & 437,5 & 276414 & 447,3 & 277000 & 2,240 & 0,212 \\
\cline { 2 - 7 } 4 & 443,7 & 298600 & 450,1 & 299100 & 1,442 & 0,167 \\
\hline
\end{tabular}

In Figure 10 the behavior of the air in the centrifugal compressor of the iSTC-21v jet engine is shown. Streamlines are showing vortices that are generated behind the stator vanes. From the picture the character of the flow can be seen, there is no significant turbulence and we can consider also the numerical results as relevant.

The presented results with the rest of the computed states are necessary for the creation of the new methodology, thus the results will be used in further research. Based on the computed temperatures and pressures error coefficient will be estimated according to the comparison with the measured temperatures and 
pressures. The coefficient will be used for the model with the water injection system, the idea is described in Chapter 3.

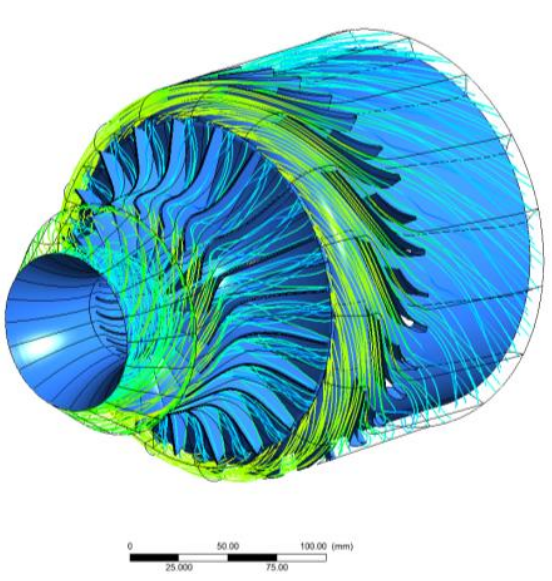

Figure 10

Streamlines of the air in the compressor

\section{Methodology for the Water Injection System Design}

The water injection into the compressor was already used for the thrust increase and multiple studies were performed, the amount of water was estimated mainly experimentally, however until nowadays there is no unified methodology for the water amount estimation. Also, the methodology for water injection which would be based on numerical models is not developed. Using obtained models from the previous chapters the method will be developed. The numerical model (Figure 2) from the previous chapters is used as one part of the scheme in Figure 11. There are some articles, in which the water injection impact on the compressor is investigated, for instance in the paper [14], the effect of the water vaporization on the compressor is investigated. In [15] are presented the results of a calculation study of motion and evaporation of water in the compressor flow path of a GT009 gas-turbine. Other articles deal with the use of water for cooling engine parts [16], but also with the water injection into the other parts as for example the combustion chamber. The impact of the water injection is tested and the results are compared in [17] for the microturbine, the results are compared in characteristic maps and running curves of the compressor, the contribution of the water on the power augmentation was proved. Also, in paper [18] is presented the water effect of the environmental conditions on the thermodynamic cycle 
processes of a gas turbine, by using analytical relations. There are many studies of the water impact of the turbine performance, the evaporation of the water, experiments, etc. [19] but there is not a methodology of the water injection, which is based on the estimation of the amount of water using CFD methods.

The main goal of the proposed article is the development of a new methodology for assessing the amount of the injected water into the compressor for a thrust increase. Injected water is usually estimated experimentally [20, 21] but in the presented article the new approach using modern tools such as CFD modeling and experimental modeling is revealed. The methodology is based on the numerical simulations that are compared with experimental results. The main idea behind the methodology is presented in Figure 11.

As was already mentioned the whole process is depended on the numerical simulation, and that is the reason why the basic element of the scheme (Figure 11) is a $3 \mathrm{D}$ model. The methodology can be divided into three sections, the first one is the CFD simulation without the water injection, the second part is the simulation with the water injection and the third one is the experiment. For both simulations (with and without water injection) the thermodynamical parameters should be estimated for multiple regimes. For particular working conditions of the compressor, the temperature and the pressure should be computed.

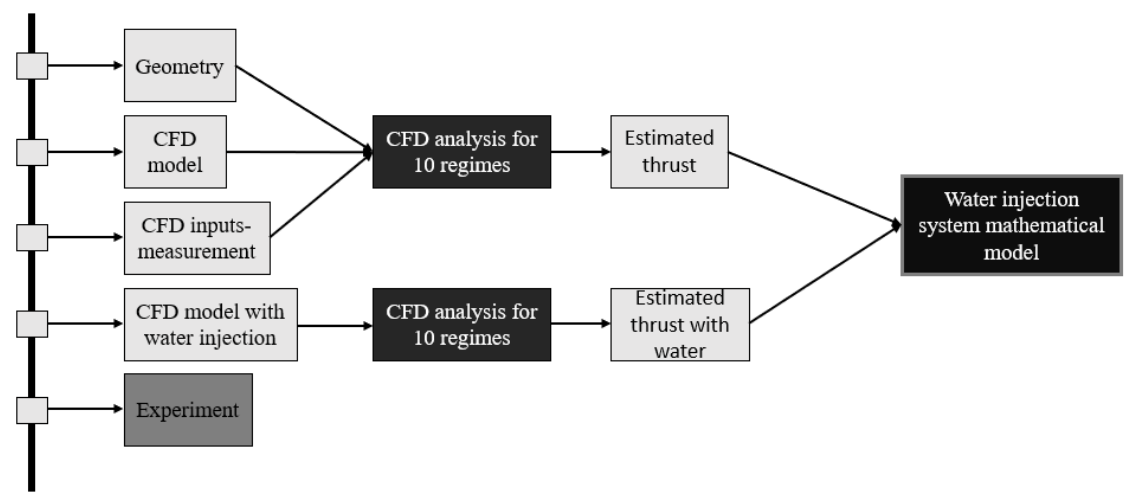

Figure 11

Methodology for the water injection system development

The computed temperature $\mathrm{T}_{2 \mathrm{t}}$ and pressure $\mathrm{p}_{2 \mathrm{t}}$ have to be compared with measurement in the real engine, so the error of CFD simulation can be estimated. It means we can assess the error coefficient by performing a sensitivity study between the CFD simulation (without water injection) and experimental data for multiple regimes.

Once the error coefficient of the CFD study is estimated it is possible to create an accurate CFD simulation of the compressor with a water injection system. It is possible to create a CFD simulation of the system for water injection and inject 
different amounts of water into the compressor (also perform few regimes the same as without the injection) and multiply the results with error coefficient [22]. Using this methodology the impact of water on the $T_{2 t}$ and $p_{2 t}$ can be obtained. Subsequently, according to the computed $\mathrm{T}_{2 \mathrm{t}}, \mathrm{p}_{2 \mathrm{t}}$ (with water injection) [23] and measured data $T_{2 t}, p_{2 t}$ it is possible to estimate the thrust of the engine [24, 25]. The described method in Figure 11 is applicable to each engine, by creating particular parts of the method for the particular engine. The method is innovative due to the fact that the amount of water is possible to estimate using numerical modeling [26, 27].

As for further research in the field, the methodology will be applied to the TKT-1 turbojet engine and according to the simulations, the water injection system will be designed. The number of the nozzles and their diameter will be estimated as well as the pressure of the water that will be injected into the compressor. The preliminary design of the system is shown in Figure 12, the methodology will be applied to the model in the following figure.
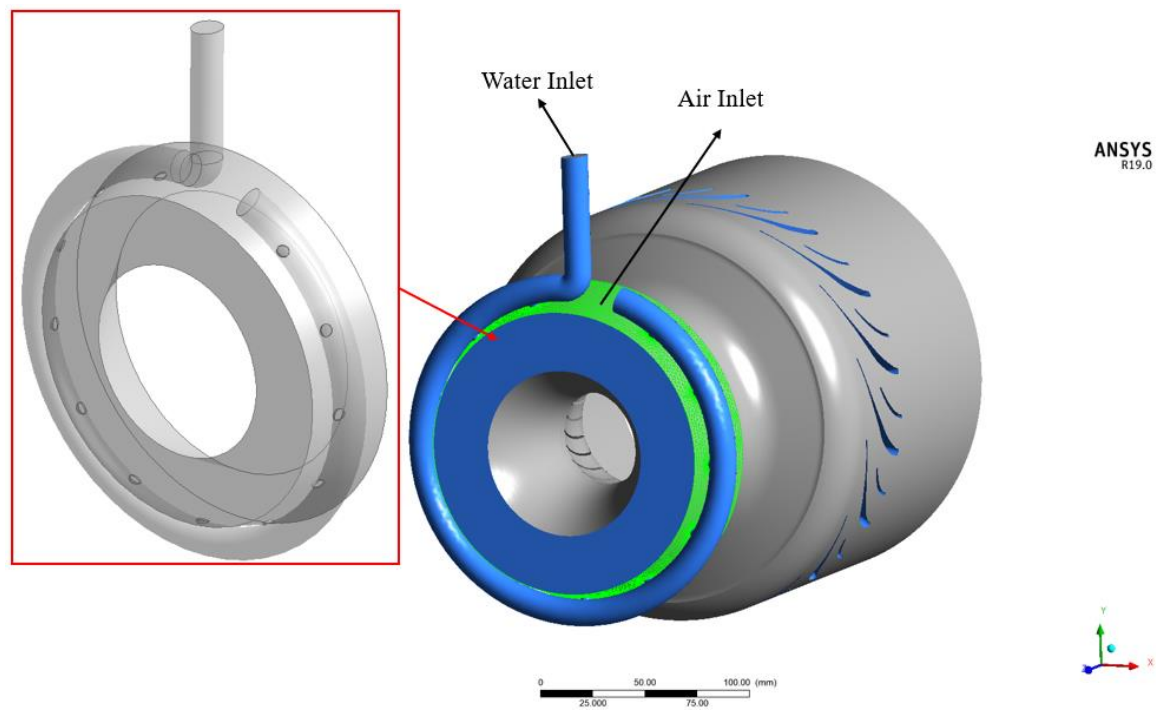

Figure 12

Preliminary design of the water injection system

\section{Conclusion}

Future research is strongly based on the proposed methodology in the article. According to the 3D CFD models, it is possible to create other analyses for the research in the field of water injection and its impact on the thermodynamic cycle of the jet engine. The CFD models created in the first part of the work are highly valuable, according to this model it will be possible to create mathematical dependence between the measured data and CFD models. 
The results presented in the paper are highly valuable for further research in terms of the water injection system. Figures 7 and 8 are showing that the results are credible due to the small deviation between the measured data in the engine iSTC$21 \mathrm{v}$ and estimated temperature and pressure using CFD analysis. The analysis was performed for the third regime and the measured pressure during this operating point is $276414,6 \mathrm{~Pa}$, estimated pressure using CFD analysis is $277000 \mathrm{~Pa}$ so the error between the analysis and measured data is $0.21 \%$. Also, data in Table 1 represents the high accuracy of the created model of iSTC-1v turbojet. Based on the results presented in the first section of the paper the error coefficient will be estimated for the model with a water injection system (Figure 12). Using this dependence, it will be possible to estimate an accurate CFD model with a water injection system.

Further research will be based mainly on the proposed methodology and the created CFD models, the preliminary idea of the water injection system is in Figure 12. The ideology is based on the tested CFD model with an added domain in front of the compressor. The domain is a preliminary water injection system. According to the methodology in Figure 11 and the model in Figure 12 the amount of water will be estimated. The simulation will be carried out for multiple regimes (Figure 5) and the results will be used for the water injection system design. The number of injection nozzles, the water pipe, water pump, etc. will be estimated according to the results.

\section{Acknowledgment}

The project was supported by KEGA 044TUKE-4/2019 - A small unmanned airplane - the platform for education in the area of intelligent avionics. And by the Hungarian National EFOP-3.6.1-16-2016-00014 project titled "Investigation and development of the disruptive technologies for e-mobility and their integration into the engineering education" (IDEA-E). The work was also support of research and development potential in the area of transport means with ITMS project code: 313011 T557

\section{References}

[1] E. Sundröm, B. Semlitsch, M. Mihaescu, Generation Mechanisms of Rotating Stall and Surge in Centrifugal Compressors, Flow, Turbulence Combustion, Vol. 100, pp. 705-719, 2018

[2] R. A. Togh, A. M. Tousi, M. Soltani, Design and CFD analysis of centrifugal compressor for a microgasturbine, Aircraft Engineering and Aerospace Technology, pp. 137-143, 2007

[3] M. T. Shobhavathy, P. Hanoca, CFD Analysis to Understand The Flow Behaviour of a Single Stage Transonic Axial Flow Compressor, Proceedings of ASMEGTINDIA, 2013

[4] M. Omidi, H. J. Liu, S. Mohtaram, H. T. Lu, H. Ch. Zhang, Improving Centrifugal Compressor Performance by Optimizing the Design of 
Impellers Using Genetic Algorithm and Computational Fluid Dynamics Methods, Sustainability, pp. 1-18, 2019

[5] B. Semlitsch, M. Micaescu, Flow Phenomena Leading to Surge in a Centrifugal Compressor, Energy, Vol. 103, pp. 572-587, 2016

[6] X. Cheng and R. S. Amano, Studz of the Flow in Centrifugal Compressor, International Journal of Fluid Machinerz and Systems, Vol. 3, pp. 260-270, 2010

[7] Z. Sun, X. Zheng, Z. Linghu, Flow Characteristics of a Pipe Diffuser for Centrifugal Compressors, Journal of Applied Fluid Mechanics, Vol. 10, No. 1, pp. 143-155, 2017

[8] A. Hafaifa, R. Belhadef, M. Guemana, Modelling of surge phenomena in a centrifugal compressor: experimental analysis for control, Systems Science \& Control Engineering An Open Access Journal, Vol. 2, pp. 631-641, 2014

[9] L. Főző, R. Andoga, K. Beneda, J. Kolesar, Effect on Operating Point Selection on Non-linear Experimental Identification of iSTC-21v and TKT1 Small Turbojet Engines, Periodica Plytechnica Transportation Engineering, pp. 141-147, 2017

[10] ANSYS CFX-Solver Theory Guide, ANSYS, Inc. Release 16.0 January 2015

[11] ANSYS CFX Tutorials, Release 15.0, ANSYS, Inc., 788 pages, 2014

[12] ANSYS TurboGrid Tutorials, ANSYS, Inc. Release 16.0, 2015

[13] I. Roumeliotis, K. Mathioudakis, Evaluation of water injection effect on compressor and engine performanc eand operability, Applied Energy 87, pp. 1207-1216, 2010

[14] A. J. White, A. J. Meacock, An Evaluation of the Effects of Water Injection on Compressor Performance, Journal of Engineering for Gas Turbines and Power, 2004, pp. 748-754, 2004

[15] Yu. M. Anurov, A. Yu. Peganov, A. V. Skvortsov, A. L. Berkovich, and V. G. Polishchuk, Calculation Study of Water Injection on Compressor Characteristics of a GT-009 Gas-Turbine Installation, Thermal Engineering, Vol. 53, pp. 964-969, 2006

[16] U. Metha, J. Bowles, J. Melton, L. Huynh, P. Hagseth, Water injection precompressor cooling assist space access, The Aeronautical Journal, Vol. 119 , pp. $145-171,2015$

[17] K. Suzuki, S. Nakano, K. Seki, Y. Tekeda, T. Kishibe, Effects of Water Injection on Generator Output Power Augumentation in a Microturbine, International Symposium on Transport Phenomena and Dynamics of Rotating Machinery, 2017 
[18] R. Kadi, A. Bouam, S. Aissani, Analzye of gas turbine performances with the presence of the steam water in the combustion chamber, Revue des Energles Renouavelables, pp. 327-335, 2007

[19] S. Schuster, D. Brillert, U Martens, V. Hermes, F. K. Benra, Investigation of the evaporation process of liquefied hzdrocarbons in front of a compressor, International Szmposium on Transport Phenomena and Dynamics of Rotating Machinerz, ISROMAC, United states, 2019

[20] T. Ous, E. Mujic, N. Stosic, Experimental investigation on -water injected twin screw compressor for fuel cell humidification, International Journal of Hzdroge, 2011

[21] M. Obermuller, K. J. Schmidt, H. Schulte, D. Peitsch, Some Aspects on Wet Compression - Physical Effects and Modeling Strategies Used in Engine Performance Tools, Deutscher Luft- und Raumfahrtkongres, 2012

[22] R. Andoga, L. Főzo, L. Madarász, T. Karol', “A Digital Diagnostic System for a Small Turbojet Engine," Acta Polytechnica Hungarica, Vol. 10, No. 4, 2013, ISSN 1785-8860

[23] E. Kiyak, A. Kahvecioglu, F. Caliskan, "Aircraft Sensor and Actuator Fault Detection, Isolation, and Accommodation," in Journal of Aerospace Engineering, Vol. 24, No. 1, 2011, pp. 47-58

[24] X. Wei, G. Yingqing, "Aircraft Engine Sensor Fault Diagnostics Based on Estimation of Engine's Health Degradation," Chinese Journal of Aeronautics, Vol. 22, No. 1, 2009, pp. 18-21, ISSN 1000-9361

[25] L. Főző, J. Judičák, R. Bréda, S. Szabo, R. Rozemberg, M. Džunda, Intelligent Situational Control of Small Turbojet Engines, Hindawi, International Journal of Aerospace Engineering, 2018

[26] M. Spodniak, M. Klimko, M. Hocko, P, Žitek, Low cycle fatigue numerical estimation of a high pressure turbine disc for the AL-31F jet engine, EPJ Web of Conferences, Vol. 143, 2017, ISSN 2101-6275, pp. 1-5

[27] Roman, R.-C., Radac, M.-B., Precup, R.-E., Petriu, E. M.., "Data-driven Model-Free Adaptive Control Tuned by Virtual Reference Feedback Tuning," Acta Polytechnica Hungarica, Vol. 13 No. 1, 2016, ISSN 17858860, DOI: 10.12700/APH.13.1.2016.1.7 